\title{
A comparative study of oblique lumbar interbody fusion combined posterior percutaneous internal fixation and the anterior-posterior combined surgery in the treatment of single-segment lumbar tuberculosis
}

Zuozhong Liu (D liuzuozhong2020@sina.com)

Yongchuan Hospital of Chongqing Medical University https://orcid.org/0000-0002-2027-7205

Lili Huang

Yongchuan Hospital of Chongqing Medical University

Jiaqin Yuan

Yongchuan Hospital of Chongqing Medical University

Gaohai Shao

Yongchuan Hospital of Chongqing Medical University

Bo Li

Yongchuan Hospital of Chongqing Medical University

Zhongliang Deng

The Second Affiliated Hospital of Chongqing Medical University

Research article

Keywords: Lumbar tuberculosis, Minimally invasive, Oblique lumber interbody fusion, Posterior percutaneous pedicle screw fixation

Posted Date: October 9th, 2020

DOI: https://doi.org/10.21203/rs.3.rs-88623/v1

License: (c) (i) This work is licensed under a Creative Commons Attribution 4.0 International License.

Read Full License 


\section{Abstract}

Objectives:To study the clinical effect of OLIF channel combined with posterior percutaneous pedicle screw fixation in the treatment of single-segment lumbar tuberculosis.

Methods:Using a retrospective controlled study, patients who were diagnosed with single-segment lumbar tuberculosis were divided into the minimal invasive group(the anterior debridement and fusion by OLIF technique combined percutaneous pedicle screw group) and the open group(the anterior-posterior combined surgery group) according to different surgical methods from July 2016 to October 2018,then the surgical-related parameters and surgical effects of the two groups of patients were compared.

Results:The minimal invasive group was superior to the open group in operation time, abdominal incision length,intraoperative blood loss,postoperative drainage, and CPK,the differences were statistically significant $(P<0.05)$; The VAS scores of low back pain after 3 days,3mounths and last follow-up of surgery was significantly lower than before surgery in both goups $(P<0.05)$, which in the minimal invasive group was significantly lower than those in the open group at 3days after surgery $\mathbb{Q P}<0.05 \rrbracket$, but no significance in other time point(Pख0.05).The ODI index, ESR, CRP before operation, 3 months and the last follow-up after surgery were not statistically different between two groups(Pख0.05), and significant difference in the group $(P<0.05)$. Postoperative lumbar lordosis increased significantly in the two groups after operation, and there was no statistical difference between the groups at each time point( $P \bowtie 0.05)$.

Conclusion:The clinical effect of OLIF channel combined with posterior percutaneous pedicle screw fixation in the treatment of single-segment lumbar tuberculosis is not different from the anterior-posterior combined surgery, but the trauma is smaller and the recovery is faster in the former, which has certain advantages in clinic.

\section{Background}

Among systemic bone and joint tuberculosis,the incidence of spinal tuberculosis is the highest,about $50 \%$, of which lumbar tuberculosis accounts for $22.8 \%-45 \%[1,2]$.Most patients can be cured with systemic nutritional support and reasonable anti-tuberculosis treatment[3]. However,in spite of adequate medical treatment,some patients'condition is still progressing,forming cold abscess,spinal instability,symptoms of spinal cord or cauda equina compression,kyphosis,etc.Surgical therapy is indicated and recommended after the above complications occur[4].

Lesion removal and spinal function reconstruction are the main goals of surgical treatment.surgical methods usually include:anterior lesion removal, bone graft fusion and internal fixation;posterior spinal canal decompression,lesion removal, and pedicle screw internal fixation;Anterior lesion removal,bone graft fusion, posterior pedicle screw internal fixation,etc[5, 6]. However,the above surgical methods have the features of large trauma and slow postoperative recovery. 
With the development and application of various techniques in spinal surgery,minimally invasive treatment of lumbar tuberculosis has become a new method.Among the various minimally invasive treatment methods,oblique lateral lumbar interbody fusion (OLIF) is a relatively popular minimally invasive anterior lumbar fusion technique, which is mainly used for degenerative diseases of the lumbar spine.And percutaneous pedicle screw fixation is the most common posterior minimally invasive internal fixation.At present,the combination of two minimally invasive techniques has not been reported in the literature for the minimally invasive treatment of lumbar tuberculosis. Therefore,we will combine two minimally invasive procedures for the treatment of single-segment lumbar tuberculosis,and compare them with the classical combined anterior and posterior surgery to evaluate the clinical effect,which is reported as follows:

\section{Methods}

\section{General information}

From July 2015 to July 2018,the patients diagnosed with single-segment lumbar tuberculosis,were divided into the minimally invasive group who were underwent OLIF channel lesion removal, bone graft fusion combined with post-percutaneous internal fixation,and open group who were underwent the classical combined anterior andposter-ior surgery, according to different surgical methods.Among them,there were 15 patients in the minimally invasive group,including 9 males and 6 females, with an average age of 45.2 years (21-67 years). There were 18 patients in the open group,including 10 males and 8 females, with an average age of 46.7 years (23-69 years). There was no statistical difference in general information between the two groups(Table 1).

Table 1 Baseline characteristics of the studies

\begin{tabular}{|c|c|c|c|}
\hline & $\begin{array}{l}\text { Min group } \\
(n=15)\end{array}$ & $\begin{array}{l}\text { Open group } \\
\quad(n=18)\end{array}$ & $\mathrm{P}$ value \\
\hline Men/ Women (n) & $9 / 6$ & $10 / 8$ & 1 \\
\hline Age $(x \pm s$, years $)$ & $45.2 \pm 9.2$ & $46.7 \pm 10.4$ & 0.676 \\
\hline Course ( $\mathrm{x} \pm \mathrm{s}, \mathrm{month})$ & $2.2 \pm 0.3$ & $2.2 \pm 0.3$ & 0.911 \\
\hline Level (cases) & & & 1 \\
\hline L2-3 & 1 & 1 & \\
\hline L3-4 & 5 & 7 & \\
\hline L4-5 & 9 & 10 & \\
\hline Frankel Grading & & & 1 \\
\hline $\mathrm{C}$ & 1 & 2 & \\
\hline $\mathrm{D}$ & 4 & 4 & \\
\hline $\mathrm{E}$ & 10 & 12 & \\
\hline VAS Score & $5.4 \pm 0.9$ & $5.3 \pm 1.0$ & 0.804 \\
\hline
\end{tabular}


Inclusion criteria:Single-segment lumbar tuberculosis from L1-L5.Bone destruction,paraspinal abscess, kyphosis or spinal instability.The lesions which were located in the anterior or middle column.

Exclusion criteria:Multi-segment tuberculosis of lumbar spine.Lumbar tuberculosis between the L5 and $\mathrm{S} 1$ segment.Huge cold paraspinal abscess. Huge abscess in spinal canal with severe nerve damage.Psoas muscle hypertrophy.Recurrent lumbar tuberculosis. The patients who combined with multiple underlying diseases. There were obvious surgical contraindications.

\section{Preoperative Preparation}

\section{Relevant auxiliary examination}

Both groups of patients were perfected before surgery:X-ray,CT(chest and lumbar spine),MRI,erythrocyte sedimentation rate (ESR) and C-reactive protein (CRP).X-ray showed vertebral instability,intervertebral stenosis and local kyphosis.CT and MRI suggested that the vertebral bone destruction did not exceed the lower $2 / 3$ of the upper vertebra and the upper $1 / 3$ of the lower vertebra,the disc destruction,the cold abscess in the perivertebral and vertebral canal.Pulmonary tuberculosis was excluded in both groups.In the minimally invasive group,the erythrocyte sedimentation rate was $72.9 \pm 10.2 \mathrm{~mm} / \mathrm{h}$, and C-reactive protein was $39.8 \pm 5.3 \mathrm{mg} / \mathrm{L}$. In the open group, erythrocyte sedimentation rate was $75.9 \pm 5.4 \mathrm{~mm} / \mathrm{h}$ and C-reactive protein was $39.7 \pm 4.5 \mathrm{mg} / \mathrm{L}$.

\section{Preoperative treatment}

All patients were told to rest in bed and take isoniazid $300 \mathrm{mg} / \mathrm{d}$, rifampicin450-600 mg/d(based on body weight), pyrazinamide $1500 \mathrm{mg} / \mathrm{d}$ and ethambutanol $750 \mathrm{mg} / \mathrm{d}$ orally for more than two weeks. High protein, high energy and other nutritional support treatment.Reexamination showed that erythrocyte sedimentation rate was lower than $50 \mathrm{~mm} / \mathrm{h}, \mathrm{C}$-reactive protein was close to normal,the functions of liver and kidney were normal.

\section{Surgical Methods}

\section{Minimally invasive group}

First,posterior minimally invasive percutaneous pedicle screw fixation was operated. After tracheal intubation general anesthesia, the patient was placed in prone position with soft pillow on chest and hip.To locate the vertebral body that needed to be fixed under C-arm fluoroscopy and mark the body surface.The paraspinal skin incisions,each approximately $1.5 \mathrm{~cm}$ in length,were made at the marked point of the body surface after disinfection in the surgical area. The puncture needle was implanted to the proper position of the vertebral body,then the needle core was pulled out,the guide wires was inserted,the sheath of the needle was removed while maintaining the position of the guide wires within the pedicle,and the pedicle screws were implanted after dilatation along the guide wires. The rods were placed with the aid of a rod guider and the nuts were screwed tight to correct the deformity and restore lordosis and stability of the lumbar spine.All steps were assisted by the C-arm. 
Second,The lateral oblique lumbar interbody fusion was performed. The patient was changed to a standard lateral position. The diseased intervertebral space was located and marked at the skin under the help of C-arm.The skin, subcutaneous, and deep fascia were cut sequentially at the lateral abdominal wall with a $7 \mathrm{~cm}$ incision along the direction of the external oblique muscle. The abdominal muscles were separated to retroperitoneum,then the psoas was pushed to posterior and the blood vessels were pushed to anterior.The guide needle was implanted into the diseased intervertebral space with the help of Carm,and the stepwise expansion tube was placed over guide needle,finally the working channel was placed,fixed and stretch to expose the surgical field.The diseased intervertebral disc,necrotic bone,granulation tissue and cheese-like material were resected,then autologous bone blocks or titanium cages with autologous bone particles were implanted into the lesion space and embedded tight. the incision was closed layer by layer after the bleeding was completely stopped and drainage tube was placed.

\section{Open group}

First,posterior open internal fixation was operated.patients were placed in the prone position after general anesthesia.The posterior midline incision was made,then the facet joints were exposed after the skin,subcutaneous and paravertebral muscles were cut in turn. The pedicle screw was implanted through the herringbone ridge under the guidance of the $\mathrm{C}$-arm,then the titanium rod was placed and the nut was tightened,so as to correct the kyphosis and restore the normal sequence.Second,The patient was changed to a lateral position. With the lesion as the center,a long oblique incision from the 12th rib to the anterior iliac was made, then the skin,subcutaneous, external oblique muscle,internal oblique muscl,transverse abdominal muscle were incised in turn. The psoas major muscle was exposed,splited longitudinally and pushed to both sides to expose the intervertebral disc of the diseased segment,the upper and lower vertebrae through the peritoneum. Then the treatment of the lesion intervertebral space and the method of closing the incision was same as the minimally invasive group.

\section{Postoperative Treatment}

Antibiotics were routinely used for 3 to 5 days to prevent infection after operation. The combined treatment of four anti-tuberculosis drugs (isoniazid,rifampicin,ethambutanol and pyrazinamide) was continued for 12 to 18 months normatively,systemic nutritional support was strengthened at the same time.The liver and kidney function, ESR,CRP were re-examined regularly.and X-ray and CT were reviewed 3 days, 1 month, 6 months and 1 year after operation.

\section{Content Of Clinical Observation}

The length of incision, intraoperative blood loss,operation time,postoperative drainage volume,postoperative CPK,the number of complications,Visual Analogue Scale (VAS),Oswestry Dability Index (ODI),ESR,CRP and segmental lordosis(SL) recovery were recorded between two groups.

\section{Statistical Method}


SPSS23 software was used for statistical analysis,quantitative data were presented as means \pm standard deviations,the one-way analysis of variance was used for intra-group comparison,the independent $t$ test or Wilcoxon rank-sum test was used for inter-group comparison, and count data was used Fisher's exact test, $P<0.05$ was statistically significant.

\section{Results}

Both groups successfully completed the operation,the minimally invasive group was superior to the open group in terms of operation time, length of abdominal incision, intraoperative blood loss,postoperative drainage volume, and $C P K$, with statistically significant differences $(P<0.05)$,as shown in Table 2.One patient in the minimally invasive group occurred hip flexion weakness after surgery,and in the open group, two patients occurred and one occurred numbness in the front of the thigh.The above symptoms disappeared at the last follow-up of the two groups. The wounds of all patients healed in the first stage without incision infection or sinus tract formation.

Table2 Surgical related parameters of two groups

\begin{tabular}{|c|c|c|c|c|c|}
\hline & $\begin{array}{l}\text { Operation } \\
\text { time } \\
(\min )\end{array}$ & $\begin{array}{l}\text { Blood loss } \\
\text { (ml) }\end{array}$ & $\begin{array}{l}\text { Incision } \\
\text { length } \\
(\mathrm{cm})\end{array}$ & $\begin{array}{l}\text { Postoperative } \\
\text { drainage } \\
\qquad(\mathrm{ml})\end{array}$ & $\begin{array}{l}\text { CPK } \\
(\mathrm{U} / \mathrm{L})\end{array}$ \\
\hline $\begin{array}{l}\text { Min } \\
\text { group } \\
(n=15)\end{array}$ & $211.1 \pm 10.6$ & $164.4 \pm 22.1$ & $7.4 \pm 0.7$ & $196.4 \pm 17.6$ & $425.8 \pm 24.8$ \\
\hline $\begin{array}{l}\text { Open } \\
\text { group } \\
(n=18)\end{array}$ & $218.7 \pm 8.2$ & $341.0 \pm 50.9$ & $17.1 \pm 1.3$ & $346.8 \pm 50.0$ & $882.5 \pm 152.8$ \\
\hline$P$ value & 0.035 & 0 & 0 & 0 & 0 \\
\hline
\end{tabular}

Patients in both groups were followed up through outpatient clinics,phone calls or WeChat.The minimally invasive group had an average follow-up time of 24 months (12 to 36 months), and the open group had an average follow-up time of 27 months (12 to 41 months).

There was no significant difference in the VAS scores of low back pain before operation between the two groups $(P>0.05)$.The scores at each postoperative time were lower than those before the operation, and the longer the follow-up time,the lower the score.The final VAS score of the minimally invasive group and the open group were both between 2-3.However,VAS score was lower in the minimally invasive group at 3 days after surgery, and the difference was statistically significant $(P<0.05)$. There was no statistical difference in scores between the two groups at 3 months after surgery and the last follow-up $(P>0.05)$. 
There were no statistically significant differences in ODI index,ESR and CRP between the minimally invasive group and the open group before, 3 months after and at the last follow-up (Pख0.05),the comparison of each point within the group was statistically significant (Pख0.05). At the last follow-up,all patients in both groups returned to their original jobs,and inflammatory indicators reflecting tuberculosis activity were reduced to the normal range. As shown in the table 3.

Table 3 Surgical effects of two groups

\begin{tabular}{lcccc}
\hline & & Min group & Open group & P value \\
\hline VAS Score & Pre-op & $5.4 \pm 0.9$ & $5.3 \pm 1.0$ & 0.352 \\
& 3d post-op & $2.6 \pm 0.5$ & $3.3 \pm 0.6$ & 0.002 \\
& 3m post-op & $1.1 \pm 0.3$ & $1.5 \pm 0.5$ & 0.857 \\
& Last follow-up & $0.5 \pm 0.5$ & $0.4 \pm 0.5$ & 0.9 \\
\hline ODI & Pre-op & $79.3 .8 \pm 3.6$ & $80.8 \pm 3.7$ & 0.235 \\
& 3m post-op & $44.9 \pm 3.1$ & $44.2 \pm 2.9$ & 0.501 \\
& Last follow-up & $25.9 \pm 3.1$ & $27.3 \pm 3.3$ & 0.24 \\
\hline ESR(mm/h) & Pre-op & $72.9 \pm 10.2$ & $75.9 \pm 5.4$ & 0.271 \\
& 3d post-op & $95.8 \pm 7.4$ & $95.7 \pm 7.2$ & 0.968 \\
& 3m post-op & $21.6 \pm 3.8$ & $22.0 \pm 3.0$ & 0.755 \\
& Last follow-up & $8.5 \pm 1.6$ & $9.0 \pm 1.1$ & 0.253 \\
\hline CRP(mg/L) & Pre-op & $39.8 \pm 5.3$ & $39.7 \pm 4.5$ & 0.953 \\
& 3d post-op & $48.7 \pm 4.5$ & $48.5 \pm 4.1$ & 0.918 \\
& 3m post-op & $5.5 \pm 0.7$ & $6.1 \pm 0.9$ & 0.097 \\
& Last follow-up & $3.9 \pm 0.9$ & $4.5 \pm 0.8$ & 0.08 \\
\hline Complications(n) & Pre-op & $-0.6 \pm 2.0$ & $-0.2 \pm 1.7$ & 0.374 \\
& Post-op & $5.3 \pm 0.6$ & $5.7 \pm 0.8$ & 0.132 \\
& 3m post-op & $4.5 \pm 0.9$ & $4.6 \pm 0.7$ & 0.636 \\
& Last follow-up & $4.1 \pm 0.8$ & $4.3 \pm 0.7$ & 0.492 \\
\hline
\end{tabular}

Pre-op means Preoperative; Post-op means Postoperative;

Lumbar segmental lordosis was defined as the angle between the upper endplate of the upper vertebra and the lower endplate of the lower vertebra.The preoperative and postoperative differences in the group were statistically significant $(P<0.05)$, and the postoperative lumbar segmental lordosis was significantly increased.There was no statistically significant difference between the two groups at each time after 
operation $(P>0.05)$. At the last follow-up,bone fusion was achieved in the intervertebral bone grafting in both groups.See Table 3 and Fig. 1 for details.

\section{Discussion}

For the spinal tuberculosis that requires surgery, the currently commonly used surgical methods include: anterior lesion removal and bone grafting,fusion internal fixation;posterior lesion removal,bone graft fusion and internal fixation;and combined anterior and posterior surgery,etc[7, 8]. However,the abovementioned surgical methods have disadvantages such as large trauma,excessive bleeding,and many postoperative complications $[9,10]$.Minimally invasive treatment of spinal diseases,including spinal tuberculosis,is the development direction of spinal surgery, which is conducive to the rapid recovery of patients after surgery and early return to society[11].Researchers have tried to use different minimally invasive methods to treat lumbar spine tuberculosis.Ying et al[12] reported that anterior small incisions combined with posterior internal fixation were used to treat lumbar spine tuberculosis.Wang et al[13] reported that the extreme lateral channel combined with percutaneous posterior pedicle screw fixation to treat similar patients.Both have achieved good clinical results. However, none of the above methods are operated through the anatomical gap, and the trauma is still large.

Oblique lumber interbody fusion (OLIF) was first reported by French doctor Silvestre in 2012.The main process is to enter the posterior peritoneum through the natural muscle space of the side abdomen,and then push the psoas major muscle backward, place the channel in the surgical section, and perform related operations. Because of its small incision,access through a natural gap, small trauma, quick postoperative recovery, and no differences in fusion from other methods, it is currently mainly applicable to lumbar degenerative diseases, including:lumbar disc herniation,lumbar segmental instability,lumbar spine Spinal stenosis,etc $[14,15,16,17]$.Percutaneous posterior minimally invasive pedicle screw fixation is currently widely used in the posterior fixation of thoracolumbar spine diseases. It has the advantages of small incision, no need to strip the paravertebral muscles, and can significantly reduce postoperative low back pain $[18,19,20,21]$. We tried to combine the two minimally invasive surgical methods for the treatment of single-segment lumbar tuberculosis.During an average of two years of follow-up after operation, all cases achieved good results.

Traditional anterior and posterior combined open surgery, both anterior and posterior incisions are long.Zhang Jialin et al. reported that the average length of incision through the anterior approach was about $15.42 \mathrm{~cm}[22]$.In the anterior approach, the external oblique, internal oblique,transverse abdominis and psoas major muscle need to be cut off, while in the posterior approach,the multiple fissure,vertical spine and facet joints need to be peeled off, resulting in large surgical incision, heavy trauma,scar hyperplasia and loss of muscle function.

In the minimally invasive group of this study,the incision of the anterior approach was about $7 \mathrm{~cm}$,and the anterior operation was performed through the muscle gap with the help of OLIF channel,without the need to cut psoas. In the posterior approach,each skin incision was about $1.5 \mathrm{~cm}$, and the operation was 
performed through the space between the multifissure and the erector spinalis,nail rods were also placed subcutaneously. Therefore, compared with the open group,the trauma was less, the muscle injury was lighter,the intraoperative operation time was shorter,and the postoperative index of CPK reflecting the degree of muscle injury was far lower than that of the open group.Due to small muscle injury and operation under channel protection,the intraoperative blood loss and postoperative drainage volume were far lower than those of the open group and the difference between the two groups was statistically significant.

There was no significant difference in VAS score,ODI index,ESR and CRP between the two groups during postoperative follow-up $(P>0.05)$. The back and leg pain was completely relieved and all patients returned to their previous jobs,both ESR and CRP dropped to normal at the last follow-up.The minimally invasive group achieved the same long and short term clinical effects as the open group.In terms of the recovery of segmental lordosis, there was no statistical difference between the two groups at each time after the operation.And at the last follow-up, all the two groups achieved intervertebral bone graft fusion.The minimally invasive group can also restore the physiological lordosis and good fusion as the open group.

In the minimally invasive group, 1 case showed hip flexion weakness after surgery; In the open group,2 patients showed hip flexion weakness and 1 patient showed numbness in the front of the thigh.No serious complications such as paralysis, massive hemorrhage, and infection occurred.There was no difference in surgical risk between the two groups $(P=0.607)$. The minimally invasive group was as safe and feasible as the open group.

However,the study still has certain limitations.our study is retrospective and take place in single medical center,the number of clinical cases is relatively small.It is not suitable for L5-S1 lesions,huge paravertebral abscesses,hypertrophy of the psoas major,multisegmental bone destruction and so on.Therefore,prospective, multi-center,large-scale clinical research and the design of related instruments specifically for tuberculosis minimally invasive surgery are still needed to further confirm the superiority of this method.

\section{Conclusion}

The clinical effect of OLIF channel combined with posterior percutaneous pedicle screw fixation in the treatment of single-segment lumbar tuberculosis is not different from the anterior-posterior combined surgery,but the trauma is smaller and the recovery is faster in the former, which has certain advantages in clinic. However, it still needs to be further verified based on the actual situation of patients and a large number of clinical practices.

\section{Abbreviations}


OLIF: Oblique lateral lumbar interbody fusion; ESR: Erythrocyte sedimentation rate; CRP: C-reactive protein; Min group: Minimally invasive group; CPK:Creatine phosphokinase; VAS Score: Visual Analogue Scale; ODI: Oswestry Dability Index; Pre-op: Preoperative; Post-op: Postoperative; SL:segmental lordosis.

\section{Declarations}

\section{Ethics approval and consent to participate}

This study obtained ethics approval and consent from the ethics committee in our hospital.

\section{Consent for publication}

Not applicable.

\section{Competing interests}

The authors declare that they have no competing interests.

\section{Funding}

None.

\section{Authors' contributions}

ZZL,ZLD designed this study.ZZL,LLH,and JQY collected the data.ZZL,GHS,BL and LLH analyzed the data.

\section{Acknowledgements}

Not applicable.

\section{References}

[1] Yao Y , Zhang H, Liu H, et al. Prognostic factors for recovery after anterior debridement/bone grafting and posterior instrumentation for lumbar spinal tuberculosis. World Neurosurg. 2017;104:660-667.

[2] Wang H, Li C, Wang J, et al. Characteristics of patients with spinal tuberculosis: seven-year experience of a teaching hospital in Southwest China. Int Orthop. 2012;36(7):1429-1434.

[3] Watts HG, Lifeso RM. Tuberculosis of bones and joints. J Bone Joint Surg Am. 1996;78(2):288-98.

[4] Tang MX, Zhang HQ, Wang YX, Guo CF, Liu JY. Treatment of spinal tuberculosis by debridement, interbody fusion and internal fixation via posterior approach only. Orthop Surg. 2016;8(1):89. 
[5]Bezer M,Kucukdurmaz F,Aydin N,et al.Tuberculous spondylitis of the lumbosacral region:long-term follow-up of patients treated by chemotherapy,transpdeicular drainage,posterior instrumentation,and fusion[J].J Spinal Discord Tech,2005,18(5):425-429.

[6]Sahoo MM,Mahapatra SK,Sethi CC,et al.Posterior-only approach surgery for fixation and decompression of thoracolumbar spinal tuberculosis:a retrospective study[J].J Spinal Discord Tech.2012,25(7):E219-223.

[7] Hassan K, Elmorshidy E. Anterior versus posterior approach in surgical treatment of tuberculous spondylodiscitis of thoracic and lumbar spine. Eur Spine J. 2016;25(4):1056-63.

[8] Liu Z, Wang X, Xu Z, et al. Two approaches for treating upper thoracic spinal tuberculosis with neurological deficits in the elderly: A retrospective case-control study. Clin Neurol Neurosurg. 2016;141:111-6.

[9] Faciszewski T, Winter RB, Lonstein JE, et al. The surgical and medical perioperative complications of anterior spinal fusion surgery in the thoracic and lumbar spine in adults. A review of 1223 procedures. Spine (Phila Pa 1976).1995;20(14):1592-9.

[10] Ballard JL, Carlson G, Chen J, et al. Anterior thoracolumbar spine exposure: critical review and analysis. Ann V asc Surg.2014;28(2):465-9.

[11]Yang X, Luo Ch, Liu LM,et I. Minimally invasive lateral lumbar intervertebral fusion versus traditional anterior approach for localized lumbar tuberculosis: a matched-pair case control study[J]. The Spine Journal,2020, 20(3):426-434.

[12] Ying XZ, Shi SY, Zheng Q, et al. Treatment of lumbar tuberculosis by mini-open anterior approach focal cleaning combined with posterior internal fixation. Med Sci Monit. 2017;23:4158-4165.

[13] Wang QY , Huang MG, Ou DQ, et al. One-stage extreme lateral interbody fusion and percutaneous pedicle screw fixation in lumbar spine tuberculosis. J Musculoskelet Neuronal Interact. 2017;17(1):450-455.

[14] Silvestre C,Mac-Thiong JM,Hilmi R,et al.Complications and Morbidities of Mini-open Anterior Retroperitoneal Lumbar Interbody Fusion:Oblique Lumbar Interbody Fusion in 179 Patients[J].Asian Spine J,2012,6(2):89-97.

[15] Zhang JF, Fan SW, Fang XQ, et al. Application of oblique lateral interbody fusion in single level lumbar disc degeneration [J]. Chinese Journal of Orthopaedics, 2017,37 (2): 80-88.

[16] Ohtori S,Orita S,Yamauchi K,et al.Mini-open Anterior Retroperitoneal Lumbar Interbody Fusion:Oblique Lateral Interbody Fusion for Lumbar Spinal Degeration Disease[J],Yonsei Med J,2015,56(4):1051-1059. 
[17] Anjarwalla NK,Morcom RK,Fraser RD.Supplementary stabilization with anterior lumbar intervertebral fusion-a radiologic review[J].Spine(Phila Pa 1976),2016,31(11):1281-1287.

[18] Bae JS,Lee SH,Kim JS,et al.Adjacent segment degeneration after lumbar interbody fusion with percutaneous pedicle screw fixation for adult low grade isthmic spondylolistheis minimum 3 years of follow-up[J].Neurosurgery,2010,67(6):1600-1607.

[19] Jan Kocis, Martin Kelbl, Tomas Kocis,et al.Percutaneous versus open pedicle screw fixation for treatment of type A thoracolumbar fractures[J]. Eur J Trauma Emerg Surg, 2020;46(1):147-152.

[20] Qinpeng Zhao,Haiping Zhang,Dingjun Hao,et al.Complications of percutaneous pedicle screw fixation in treating thoracolumbar and lumbar fracture[J].Medicine,2018,97(29):001-006.

[21] Tamer Orief, Mohammad Alfawareh, Mohammad Halawani,et al.Accuracy of percutaneous pedicle screw insertion in spinal fixation of traumatic thoracic and lumbar spine fractures[J].Surg Neurol Int,2018,9:78.

[22] Zhang YJ,Qiao YD,Yuan HF,et al.Oblique lumbar interbody fusion combined posterior instrumentation through Wiltse approach for single segmental lumbar tuberculosis[J]. Orthopedic Journal of China, 2019,27 (21): 1954-1958.

\section{Figures}

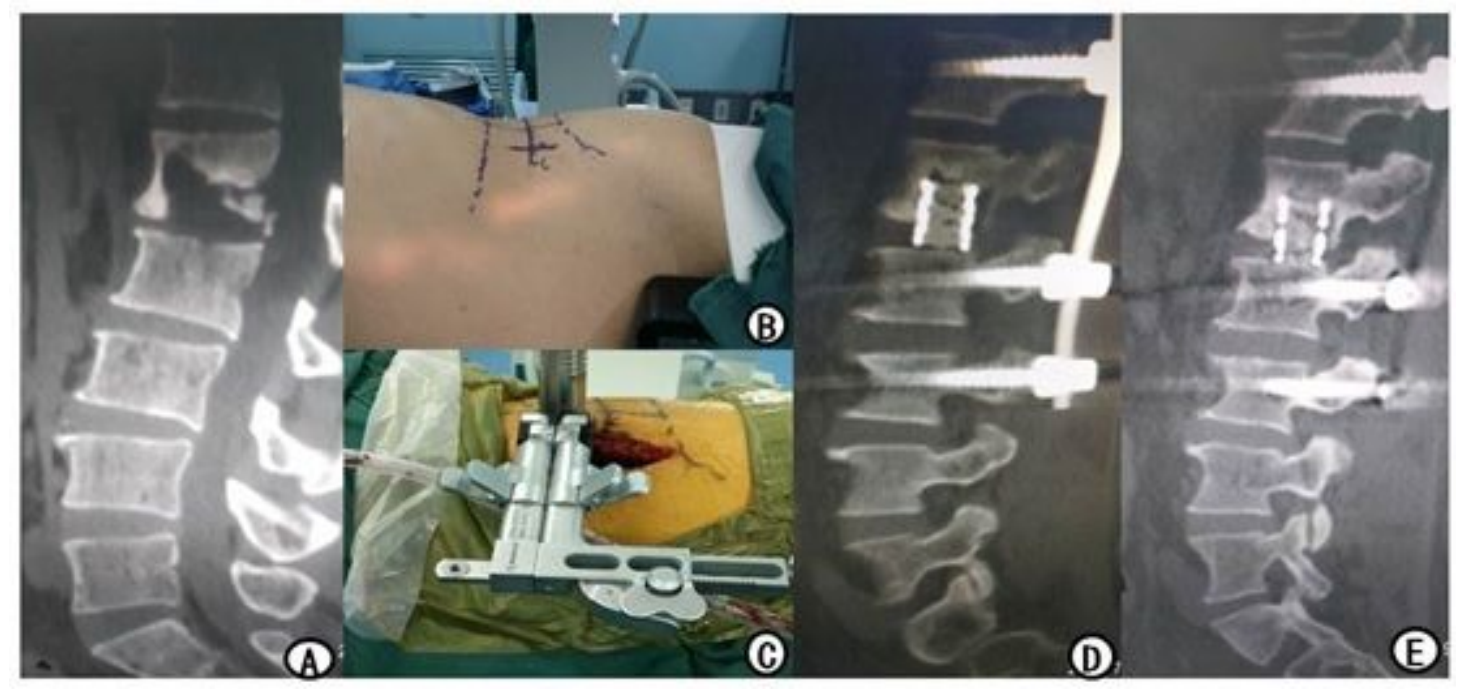

Figure 1

a patient with lumbar 1-2 tuberculosis was treated by oblique lateral intervertebral fusion combined with posterior percutaneous internal fixation. A 55-year-old male patient with L1-2 tuberculosis who received OLIF combined posterior percutaneous pedicle screw fixation.A:Preoperative CT showed L1-2 vertebrae were destructed.B:A 7-cm operation incision in the left anterolateral side of the abdomen.C:The OLIF work 
channel was settled the target segment.D:The CT scan showed theinternal fixation was reliable after 3 days of surgery.E: The CT scan showed a solid bone fusion has been achieved after 1 year of surgery. 